\title{
Supporting Information Natural Berberine-Based Chinese Herb Medicine Assembled Nanostructures with Modified Antibacterial Application
}

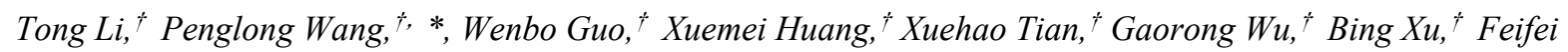
$\mathrm{Li}^{\dagger}{ }^{\dagger}$ Cong Yan, ${ }^{+}$Xing-Jie Liang, ${ }^{\xi}, *$, Haimin Lei ${ }^{\dagger}, *$

${ }^{\dagger}$ School of Chinese Pharmacy, Beijing University of Chinese Medicine, Beijing 102488, P. R. China

$\$$ School of Life Science, Beijing University of Chinese Medicine, Beijing 102488, P. R. China

$\S$ Chinese Academy of Sciences (CAS) Key Laboratory for Biological Effects of Nanomaterials and Nanosafety, CAS Center for Excellence in Nanoscience, National Center for Nanoscience and Technology, Chinese Academy of Sciences, 100190 Beijing, P. R. China

* To whom correspondence should be addressed: Penglong Wang: wp1581@126.com;

Haimin Lei: hm_lei@126.com; Xing-Jie Liang: liangxj@nanoctr.cn;

Determination of Minimum Gel Concentration. All the monomer substances (BBR, BA and WOG) in experiment were purchased from Sigma-Aldrich with their purity over $98 \%$. BA and WOG are soluble in alkalescent solution which was adjusted by $5 \% \mathrm{NaOH}$ aqueous solution to $\mathrm{pH}=8$. To determine the minimum gelation concentration, we weighted equimolar amounts of flavonoid BA, WOG and BBR, respectively. Aqueous solution with concentrations gradient from $20 \mathrm{mM}$ to $2.5 \mathrm{mM}(20 \mathrm{mM}, 15 \mathrm{mM}, 10 \mathrm{mM}, 7.5 \mathrm{mM}, 5 \mathrm{mM}$, $2.5 \mathrm{mM}$ ) were prepared. Thereafter, mixed the aqueous solution of flavonoid glycosides and BBR in the ratio of $1: 1$ under $80^{\circ} \mathrm{C}$. After cooling to room temperature, we determined that the minimum gel concentration of BA-BBR NPs was $10 \mathrm{mM}$. However, WOG-BBR NFs could not form gel at any concentration. 
FESEM Analysis of Self-Assemblies. We prepared BA-BBR NPs and WOG-BBR NFs aqueous solutions in the concentration of $0.1 \mathrm{mM}$, respectively. Then carefully pipetted $10 \mu \mathrm{L}$ and dripped on the conducting resin. The samples were dried in vacuum at room temperature, subsequently coated with gold film using a LEICA-EM-ACE600 sputter coater instrument (Leica, Germany). Finally the morphology of the self-assemblies were imaged on a FESEM (ZEISS-SUPRA55, Germany) operated at $8 \mathrm{kV}$.

TEM Analysis of Self-Assemblies. The preparation method of self-assemblies for TEM was similar with SEM. Sample solutions were drop-coated onto a carbon coated copper grid (200 mesh). After 0.5 min's adsorption, the excess was drawn off using filter paper and allowed to stand for another $20 \mathrm{~min}$. Then the samples were further dried under vacuum for $12 \mathrm{~h}$. TEM images were taken at an accelerating voltage of $100 \mathrm{kV}$ using a JEM 2100F (JOEL, Tokyo, Japan).

Particle Size Measurement. We prepared BA-BBR NPs aqueous solutions in the concentration of $0.1 \mathrm{mM}$. Particle size were determined by dynamic light scattering (DLS; Zetasizer Nano ZS 90, Malvern Instrument, UK) at $25^{\circ} \mathrm{C}$.

Ultraviolet-Visible Spectroscopy (UV). We prepared the $0.02 \mathrm{mM}$ BBR, two flavonoid glycoside sodium salt aqueous solution, as well as two self-assemblies aqueous solution, respectively. The UV spectra of samples were determined using a UV-visible Spectrophotometer (HITACHI UH5300, Japan) with the scanning range from 200 to $600 \mathrm{~nm}$.

Fourier Transform Infrared Spectroscopy (FT-IR). A FT-IR spectrometer (Nicolet iS10, Thermo, US) was used to obtain the IR spectra in the range from 4000 to $400 \mathrm{~cm}^{-1}$ by using of the $\mathrm{KBr}$ method. We compared the lyophilized powder of two self-assemblies and their monomers, respectively.

Fluorescence Emission Spectrum. Steady-state fluorescence spectra of self-assemblies were measured on a LS-45 fluorescence spectrophotometer (PerkinElmer, UK) in the range from 450 to $650 \mathrm{~nm}$. The excitation wavelength was set at $350 \mathrm{~nm}$, the scanning rate was set 
at $80 \mathrm{~nm} / \mathrm{min}$. All tests were carried out at $25{ }^{\circ} \mathrm{C}$. All spectra were run on air equilibrated solutions. Sample solutions were prepared as described in UV method.

ESI-MS Spectrum. ESI-MS was tested on a Static Spray-HRMS (Waters, US). The test results were carried out in the negative ion mode. For valuable quasi-molecular ion peaks, Secondary Mass Spectrometry of them were further determined.

Proton Nuclear Magnetic Resonance Spectroscopy of ${ }^{1}$ H-NMR. ${ }^{1} \mathrm{H}-\mathrm{NMR}$ spectra was recorded on an Avance IIIHD $400 \mathrm{MHz}$ spectrometer (Bruker, America) with tetramethylsilane as an internal standard. Monomer components $(10 \mathrm{mg})$ and lyophilized powder of self-assemblies $(15 \mathrm{mg})$ were dissolved by $1 \mathrm{~mL}$ DMSO- $d_{6}$. The ${ }^{1} \mathrm{H}-\mathrm{NMR}$ of self-assemblies were analyzed by referring the ${ }^{1} \mathrm{H}-\mathrm{NMR}$ of monomer compounds.

BA ${ }^{1} \mathrm{HNMR}\left(400 \mathrm{MHz}, \mathrm{DMSO}-d_{6}\right): \delta(\mathrm{ppm}) 6.94(\mathrm{~s}, 1 \mathrm{H}, \mathrm{H}-3), 6.99$ (s, 1H, H-8), 8.01 (d, $\left.J=6.6 \mathrm{~Hz}, 2 \mathrm{H}, \mathrm{H}-2^{\prime}, 6^{\prime}\right), 7.54-7.53\left(\mathrm{~m}, 3 \mathrm{H}, \mathrm{H}-3^{\prime}, 4^{\prime}, 5^{\prime}\right), 12.53(\mathrm{~s}, 1 \mathrm{H}, 5-\mathrm{OH}), 8.61(\mathrm{~s}, 1 \mathrm{H}$, 6-OH), $5.19\left(\mathrm{~d}, J=7.0 \mathrm{~Hz}, 1 \mathrm{H}, \mathrm{BA} \mathrm{H}-1^{\prime \prime}\right), 3.30-3.40$ (m, 3H, H-2", 3", 4"), 4.01 (d, $J=9.4$ $\left.\mathrm{Hz}, 1 \mathrm{H}, \mathrm{H}-5^{\prime \prime}\right)$.

WOG ${ }^{1} \mathrm{HNMR}\left(400 \mathrm{MHz}, \mathrm{DMSO}-d_{6}\right): \delta$ (ppm) 6.73 (s, 1H, H-3), 7.08 (s, 1H, H-6), 3.90 (s, 3H, 8- $\left.\mathrm{OCH}_{3}\right), 8.10\left(\mathrm{~d}, J=6.9 \mathrm{~Hz}, 2 \mathrm{H}, \mathrm{H}-2^{\prime}, 6^{\prime}\right), 7.64-7.62\left(\mathrm{~m}, 3 \mathrm{H}, \mathrm{H}-3^{\prime}, 4^{\prime}, 5^{\prime}\right), 12.55$ (s, $1 \mathrm{H}, 5-\mathrm{OH}), 5.30\left(\mathrm{~d}, J=5.5 \mathrm{~Hz}, 1 \mathrm{H}, \mathrm{H}-1^{\prime \prime}\right), 3.35-3.46\left(\mathrm{~m}, 3 \mathrm{H}, \mathrm{H}-2^{\prime \prime}, 3^{\prime \prime}, 4^{\prime \prime}\right), 4.05(\mathrm{~d}, J=9.4$ $\left.\mathrm{Hz}, 1 \mathrm{H}, \mathrm{H}-5^{\prime \prime}\right)$.

BBR ${ }^{1} \mathrm{H}$ NMR (400 MHz, DMSO- $\left.d_{6}\right): \delta(\mathrm{ppm}) 7.80(\mathrm{~s}, 1 \mathrm{H}, \mathrm{H}-1), 7.09$ (s, 1H, H-4), 3.21 (brs, 2H, 5- $\mathrm{CH}_{2^{-}}$), 4.96 (brs, 2H, 6- $\mathrm{CH}_{2^{-}}$), 9.92 (s, 1H, H-8), 8.20 (d, J=8.0 Hz, 1H, H-11), $8.01(\mathrm{~d}, J=8.0 \mathrm{~Hz}, 1 \mathrm{H}, \mathrm{H}-12), 8.98(\mathrm{~s}, 1 \mathrm{H}, \mathrm{H}-13), 6.18\left(\mathrm{~s}, 2 \mathrm{H}, 15-\mathrm{CH}_{2^{-}}\right), 4.10(\mathrm{~s}, 3 \mathrm{H}$, 9- $\left.\mathrm{OCH}_{3}\right)$, , $4.07\left(\mathrm{~s}, 3 \mathrm{H}, 10-\mathrm{OCH}_{3}\right)$.

BA-BBR NPs ${ }^{1} \mathrm{H}$ NMR (400 MHz, DMSO- $d_{6}$ ): $\delta$ (ppm) 6.93 (overlap, 2H, H-3,8, BA), $8.03\left(\mathrm{~d}, J=7.2 \mathrm{~Hz}, 1 \mathrm{H}, \mathrm{H}-2^{\prime}, 6^{\prime}, \mathrm{BA}\right), 7.55-7.62\left(\mathrm{~m}, 3 \mathrm{H}, \mathrm{H}-3^{\prime}, 4^{\prime}, 5^{\prime}, \mathrm{BA}\right), 4.97$ (d, $J=5.1 \mathrm{~Hz}$, 1H, H-1", BA), 3.23-3.36 (m, 3H, H-2", 3", 4", BA), 3.60 (d, J=9.6 Hz, 1H, H-5", BA), 7.74 (s, 1H, H-1, BBR), 7.02 (s, 1H, H-4, BBR), 3.19 (m, 2H, 5- $\left.\mathrm{CH}_{2^{-}}, \mathrm{BBR}\right), 4.93$ (m, 2H, 6- $\mathrm{CH}_{2^{-}}$, 
BBR), 9.94 (s, 1H, H-8, BBR), 8.08 (d, $J=9.0 \mathrm{~Hz}, 1 \mathrm{H}, \mathrm{H}-11, \mathrm{BBR}), 7.93$ (d, $J=9.0 \mathrm{~Hz}, 1 \mathrm{H}$, H-12, BBR), 8.90 (s, 1H, H-13, BBR), 6.14 (s, 2H, 15- $\left.\mathrm{CH}_{2^{-}}, \mathrm{BBR}\right), 4.07$ (s, 3H, 9-OCH BBR), 3.97 (s, 3H, 10- $\left.\mathrm{OCH}_{3}, \mathrm{BBR}\right)$.

WOG-BBR NFs ${ }^{1} \mathrm{HNMR}$ (400 MHz, DMSO-d ${ }_{6}$ ): 6.65 (s, 1H, H-6, WOG), 7.05 (s, 1H, H-3, WOG), 8.09 (d, $J=7.4$ Hz, 2H, H-2', 6', WOG), 7.64-7.62 (m, 3H, H-3', 4', 5', WOG), 3.89 (s, 3H, 8-OCH 3 , WOG), 12.48 (s, 1H, 5-OH, WOG), 5.03 (overlap, 1H, H-1", WOG), 3.15-3.29 (m, 3H, H-2", 3", 4", WOG), 3.47 (d, $J=9.7 \mathrm{~Hz}, 1 \mathrm{H}, \mathrm{H}-5 "$ ", WOG), 7.78 (s, 1H, H-1, BBR), 7.08 (s, 1H, H-4, BBR), 3.20 (brs, 2H, 5- $\left.\mathrm{CH}_{2^{-}}, \mathrm{BBR}\right), 4.93$ (m, 2H, 6- $\left.\mathrm{CH}_{2^{-}}, \mathrm{BBR}\right)$, 9.91 (s, 1H, H-8, BBR), 8.19 (d, $J=9.1 \mathrm{~Hz}, 1 \mathrm{H}, \mathrm{H}-11, \mathrm{BBR}), 7.97$ (d, $J=9.1 \mathrm{~Hz}, 1 \mathrm{H}, \mathrm{H}-12$, BBR), 8.92 (s, 1H, H-13, BBR), 6.17 (s, 2H, 15- $\left.\mathrm{CH}_{2-}, \mathrm{BBR}\right), 4.09$ (s, 3H, 9- $\left.\mathrm{OCH}_{3}, \mathrm{BBR}\right)$, $4.07\left(\mathrm{~s}, 3 \mathrm{H}, 10-\mathrm{OCH}_{3}, \mathrm{BBR}\right)$.

Table S1. ${ }^{1}$ H-NMR Chemical Shifts of Flavonoid Glycosides in Self-Assemblies

\begin{tabular}{ccccccc}
\hline Position $(\mathrm{ppm})$ & BA & BA-BBR NPs & $\Delta \delta$ & WOG & WOG-BBR NFs & $\Delta \delta$ \\
\hline H-2', 6' & 8.01 & 8.03 & 0.02 & 8.10 & 8.09 & -0.01 \\
H-3', 4', 5' & $7.54-7.53$ & $7.55-7.62$ & peak shape change & $7.64-7.62$ & $7.64-7.62$ & 0.00 \\
H-1' & 5.19 & 4.97 & -0.22 & 5.30 & 5.03 & -0.27 \\
H-5' & 4.01 & 3.60 & -0.41 & 4.05 & 3.47 & -0.58 \\
\hline
\end{tabular}

Table S2. ${ }^{1}$ H-NMR Chemical Shifts of BBR in Self-Assemblies

\begin{tabular}{cccccc}
\hline Position $(\mathrm{ppm})$ & BBR & BA-BBR NPs & $\Delta \delta$ & WOG-BBR NFs & $\Delta \delta$ \\
\hline H-1 & 7.80 & 7.74 & -0.06 & 7.78 & -0.02 \\
H-4 & 7.09 & 7.02 & -0.07 & 7.08 & -0.01 \\
H-11 & 8.20 & 8.08 & -0.12 & 8.19 & -0.01 \\
H-12 & 8.01 & 7.93 & -0.08 & 7.97 & -0.04 \\
H-13 & 8.98 & 8.90 & -0.08 & 8.92 & -0.06 \\
$10-\mathrm{OCH}_{3}$ & 4.07 & 3.97 & -0.10 & 4.07 & 0.00 \\
\hline
\end{tabular}

ROESY 2D NMR Spectrum. ROESY 2D NMR spectrum was used to detect intermolecular nuclear overhauser effects of self-assemblies. The ROESY 2D spectrum was collected with mixing time of $500 \mathrm{~ms}$ under the spin lock condition using Avance IIIHD 700 MHz Spectrometer (Bruker, America). 
Table S3. The Noteworthy Cross-Peaks in ROESY 2D NMR Spectra of BA-BBR NPs

\begin{tabular}{ccc}
\hline Cross-peak & Chemical Shifts (ppm) & Ascription \\
\hline a & $(8.03,4.07)$ & BA H-2', 6' and BBR 9-OCH $_{3}$ \\
b & $(6.93,4.97)$ & BA H-8 and BA H-1" \\
c & $(6.93,3.60)$ & BA H-8 and BA H-5" \\
\hline
\end{tabular}

Table S4. The Noteworthy Cross-Peaks in ROESY 2D NMR Spectra of WOG-BBR NFs

\begin{tabular}{ccc}
\hline Cross-peak & Chemical Shifts $(\mathrm{ppm})$ & Ascription \\
\hline $\mathrm{a}$ & $(8.09,3.89)$ & WOG H-2', 6' and WOG 8-OCH \\
$\mathrm{b}$ & $(6.65,3.47)$ & WOG H-6 and WOG H-5" \\
$\mathrm{c}$ & $(6.65,5.03)$ & WOG H-6 and WOG H-1" \\
\hline
\end{tabular}

Table S5. Energy changes data for the interactions obtained from ITC

\begin{tabular}{|c|c|c|c|c|c|c|}
\hline Number & $\begin{array}{c}\text { BA to BBR } \\
(\mu \mathrm{J})\end{array}$ & $\begin{array}{c}\text { BA to water } \\
(\mu \mathrm{J})\end{array}$ & Correction $(\mu \mathrm{J})$ & $\begin{array}{l}\text { WOG to } \\
\text { BBR }(\mu J)\end{array}$ & $\begin{array}{c}\text { WOG to } \\
\text { water }(\mu \mathrm{J})\end{array}$ & Correction $(\mu \mathrm{J})$ \\
\hline 1 & -87.28 & 11.18 & -98.46 & -58.86 & 32.3 & -91.16 \\
\hline 2 & -87.30 & 18.86 & -106.20 & -61.44 & 29.05 & -90.49 \\
\hline 3 & -105.60 & 20.14 & -125.70 & -58.29 & 24.57 & -82.85 \\
\hline 4 & -107.60 & 13.61 & -121.20 & -54.95 & 21.59 & -76.54 \\
\hline 5 & -97.75 & 13.17 & -110.90 & -50.67 & 18.7 & -69.36 \\
\hline 6 & -93.27 & 10.59 & -103.90 & -47.2 & 15.82 & -63.02 \\
\hline 7 & -81.31 & 8.16 & -89.47 & -43.38 & 12.96 & -56.34 \\
\hline 8 & -72.93 & 7.86 & -80.79 & -39.09 & 11.09 & -50.19 \\
\hline 9 & -62.85 & 7.05 & -69.90 & -36.27 & 8.694 & -44.96 \\
\hline 10 & -54.37 & 5.66 & -60.03 & -32.67 & 6.572 & -39.24 \\
\hline 11 & -43.99 & 4.66 & -48.64 & -30.53 & 5.39 & -35.92 \\
\hline 12 & -37.20 & 4.28 & -41.48 & -28.05 & 4.853 & -32.9 \\
\hline 13 & -29.58 & 3.51 & -33.09 & -28.52 & 3.839 & -32.36 \\
\hline 14 & -23.97 & 3.97 & -27.94 & -26.15 & 2.413 & -28.56 \\
\hline 15 & -18.25 & 3.35 & -21.60 & -28.09 & 2.449 & -30.54 \\
\hline 16 & -14.81 & 2.32 & -17.13 & -25.15 & 1.656 & -26.81 \\
\hline 17 & -14.46 & 2.35 & -16.81 & -24.68 & 0.9956 & -25.68 \\
\hline 18 & -9.81 & 1.97 & -11.78 & -22.6 & 0.1214 & -22.72 \\
\hline 19 & -7.00 & 2.03 & -9.03 & -20.76 & 0.5533 & -21.32 \\
\hline 20 & -6.81 & -20.20 & -6.61 & -19.34 & -0.8255 & -18.52 \\
\hline
\end{tabular}

\section{Antibacterial Assays}

The freeze-dried self-assemblies were dissolved with dimethylsulfoxide (DMSO) and nutrient broth in the ratio of $1: 2$ to a concentration of $10 \mu \mathrm{mol} / \mathrm{mL}$, then sample solution was added to the first well of a 24-well plate and diluted with nutrient broth to $0.2 \mu \mathrm{mol} / \mathrm{mL}$. Preparation methods of other concentrations were the same. 
Table S6. The inhibition rate (\%) of BBR, BA, WOG and self-assemblies on S. aureus.

\begin{tabular}{cccccc}
\hline $\mathrm{C}(\mu \mathrm{mol} / \mathrm{mL})$ & BBR & BA & WOG & BA-BBR NPs & WOG-BBR NFs \\
\hline 0.025 & $14.81 \pm 8.12$ & $2.12 \pm 8.14$ & $3.79 \pm 6.71$ & $50.85 \pm 6.03$ & $10.83 \pm 8.33$ \\
0.0375 & $45.87 \pm 4.92$ & $3.54 \pm 6.99$ & $5.83 \pm 5.98$ & $67.23 \pm 6.00$ & $32.34 \pm 6.84$ \\
0.05 & $77.78 \pm 5.34$ & $4.81 \pm 4.77$ & $7.68 \pm 5.01$ & $96.30 \pm 1.31$ & $39.32 \pm 6.88$ \\
0.075 & $88.03 \pm 5.13$ & $6.27 \pm 5.53$ & $9.90 \pm 4.43$ & $97.01 \pm 1.28$ & $43.02 \pm 4.95$ \\
0.1 & $90.31 \pm 4.04$ & $8.03 \pm 3.33$ & $12.65 \pm 3.79$ & $100.00 \pm 0.00$ & $47.15 \pm 4.53$ \\
0.15 & $100.00 \pm 0.00$ & $10.43 \pm 3.88$ & $16.79 \pm 1.25$ & $100.00 \pm 0.00$ & $53.13 \pm 4.45$ \\
0.2 & $100.00 \pm 0.00$ & $17.32 \pm 2.73$ & $19.12 \pm 2.58$ & $100.00 \pm 0.00$ & $69.23 \pm 4.76$ \\
\hline
\end{tabular}
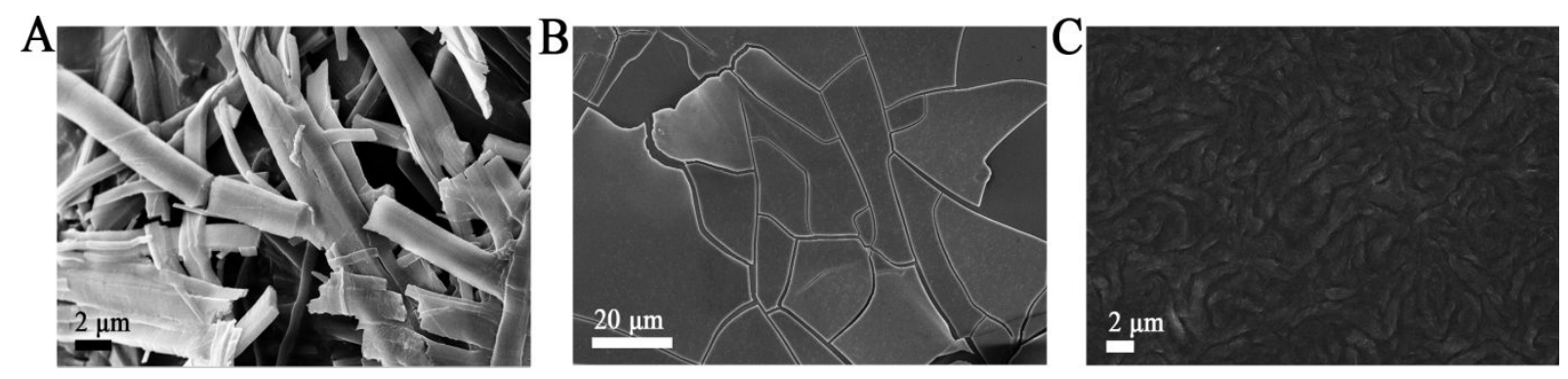

Figure S1. FESEM images of BBR (A), BA (B) and WOG (C).

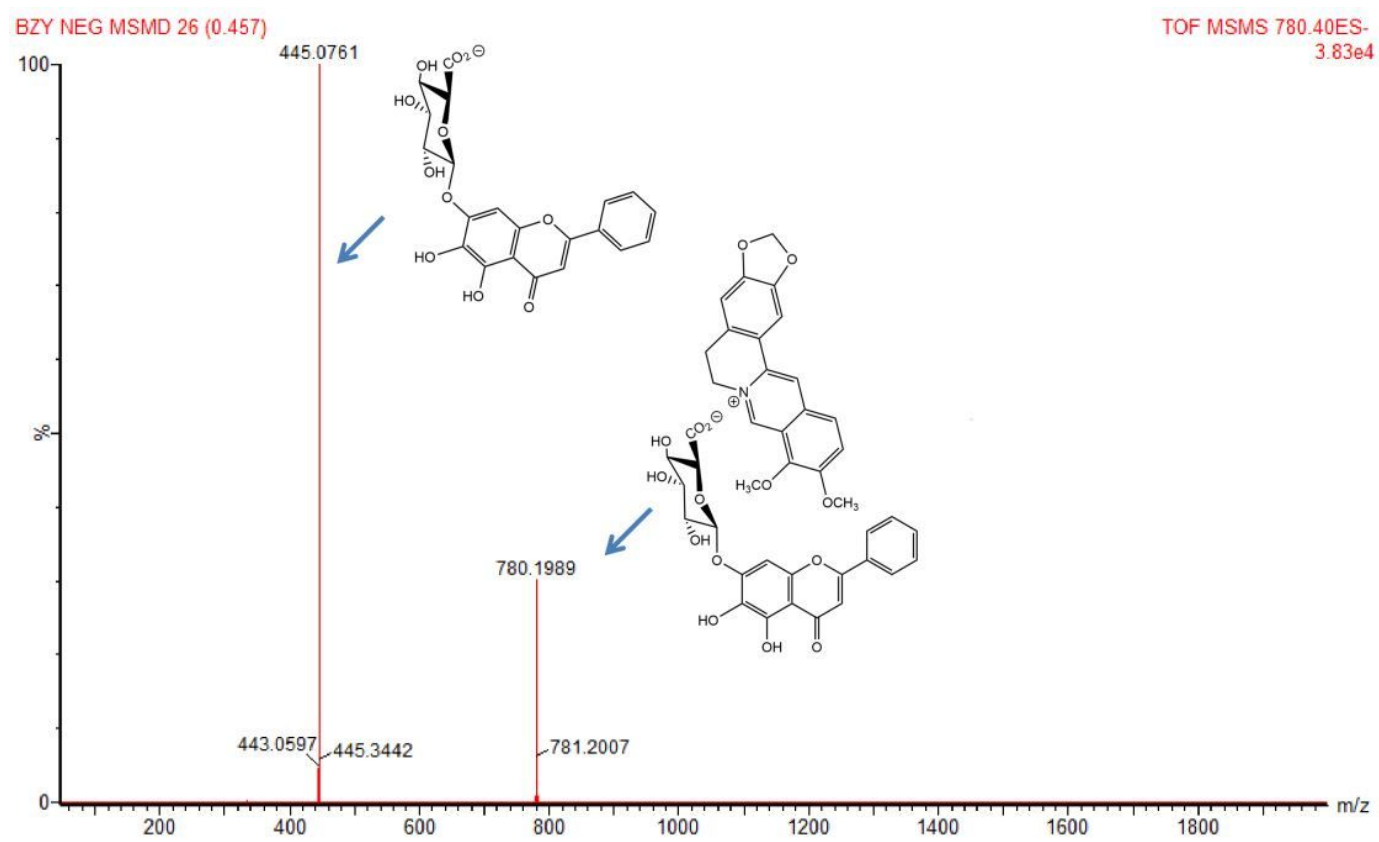

Figure S2. Secondary debris ions of BA-BBR NPs. 


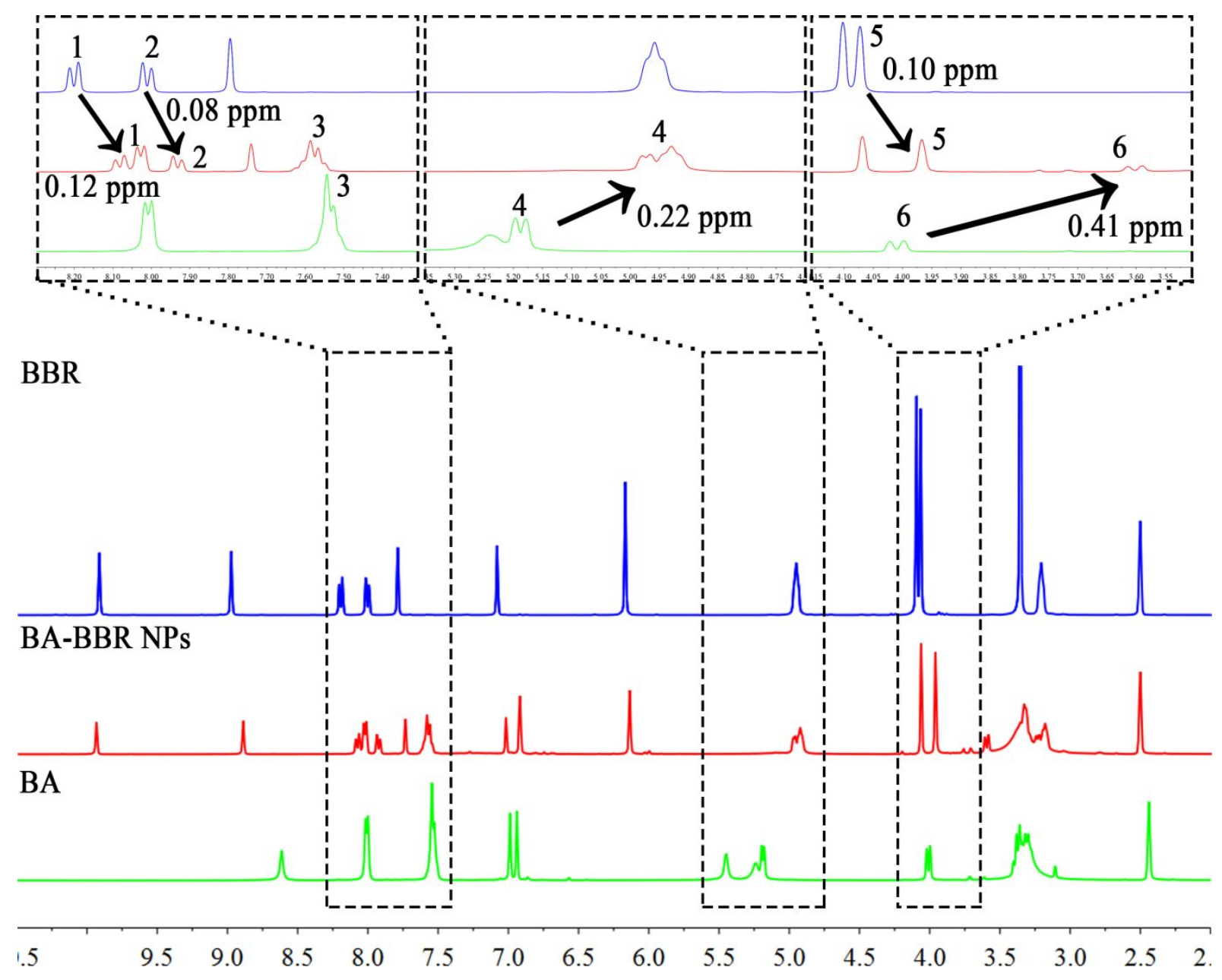

Figure S3. ${ }^{1} \mathrm{H}-\mathrm{NMR}$ spectrum of BA-BBR NPs. Peak 1 was H-11 of BBR; peak 2 was H-12 of BBR; peak 3 was H-3', 4', 5' of BA; Peak 4 was H-1" of BA; Peak 5 was $10-\mathrm{OCH}_{3}$ of BBR; Peak 6 was H-5" of BA. 


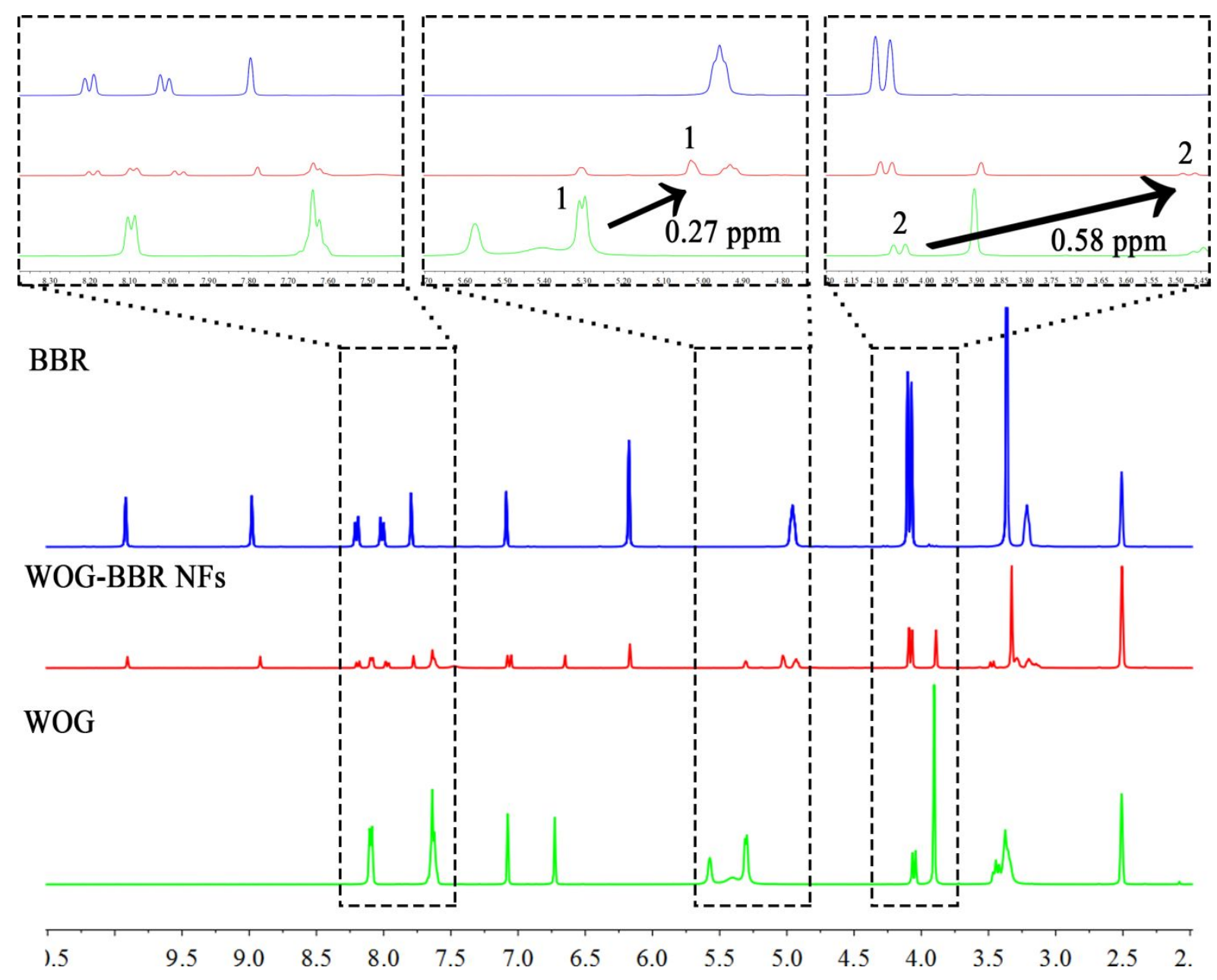

Figure S4. ${ }^{1} \mathrm{H}-\mathrm{NMR}$ spectrum of WOG-BBR NFs. Peak 1 was H-1" of WOG; Peak 2 was $\mathrm{H}-5$ " of WOG. 

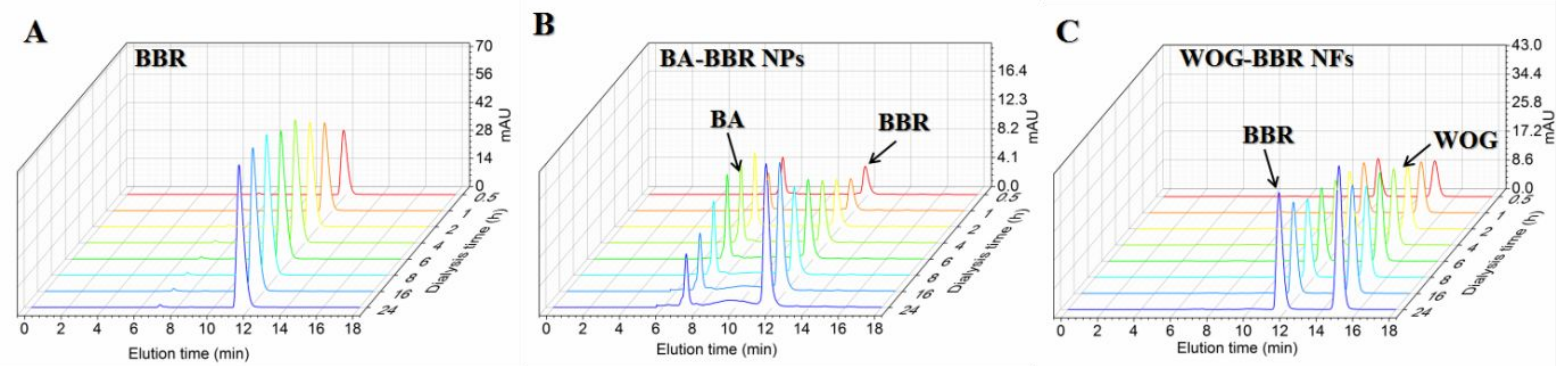

Figure S5. The drug release tests of samples. (BBR release of samples at different time by HPLC). 


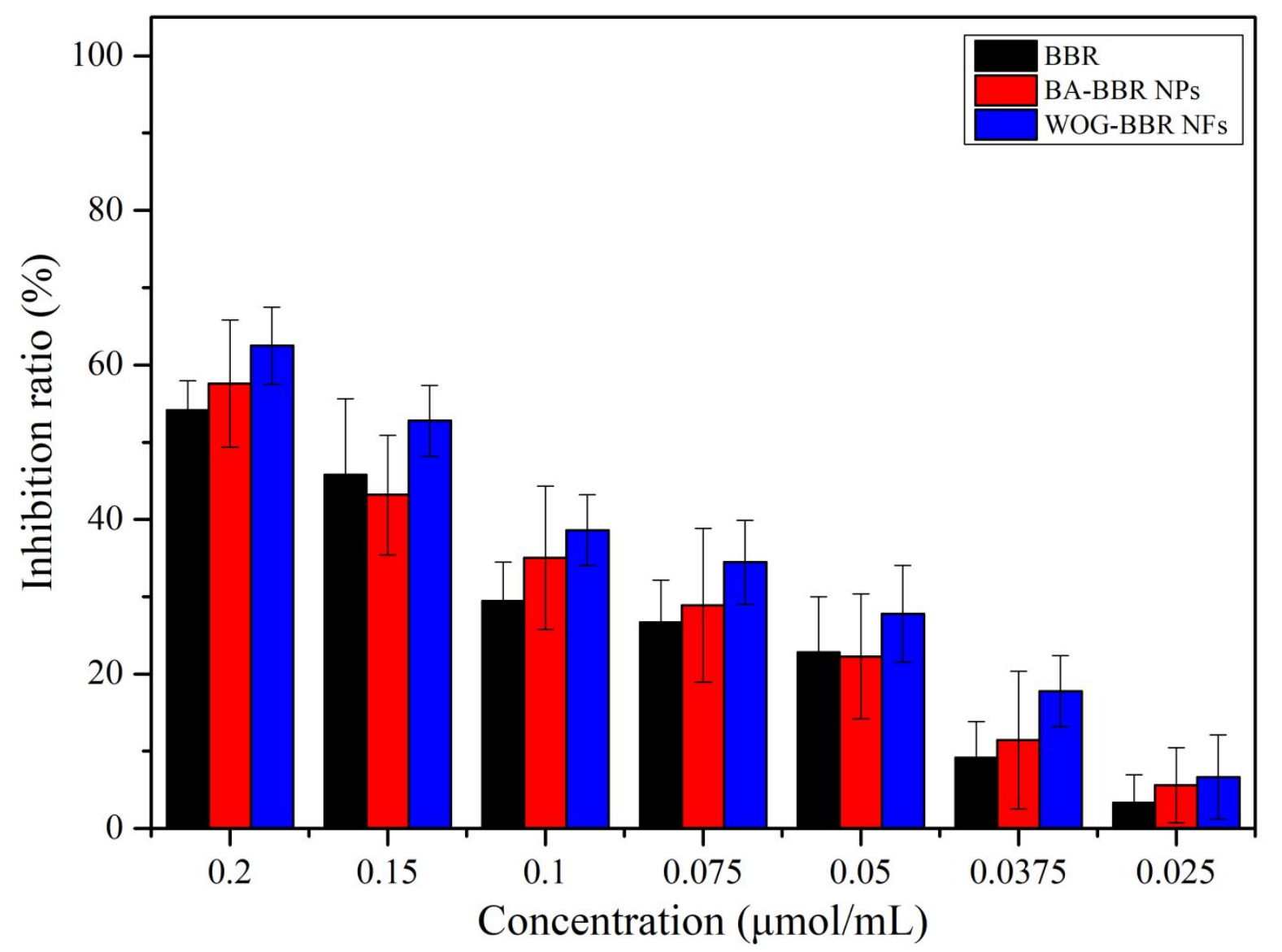

Figure S6. The inhibition ratio of BBR, BA-BBR NPs and WOG-BBR NFs on E. coli. 

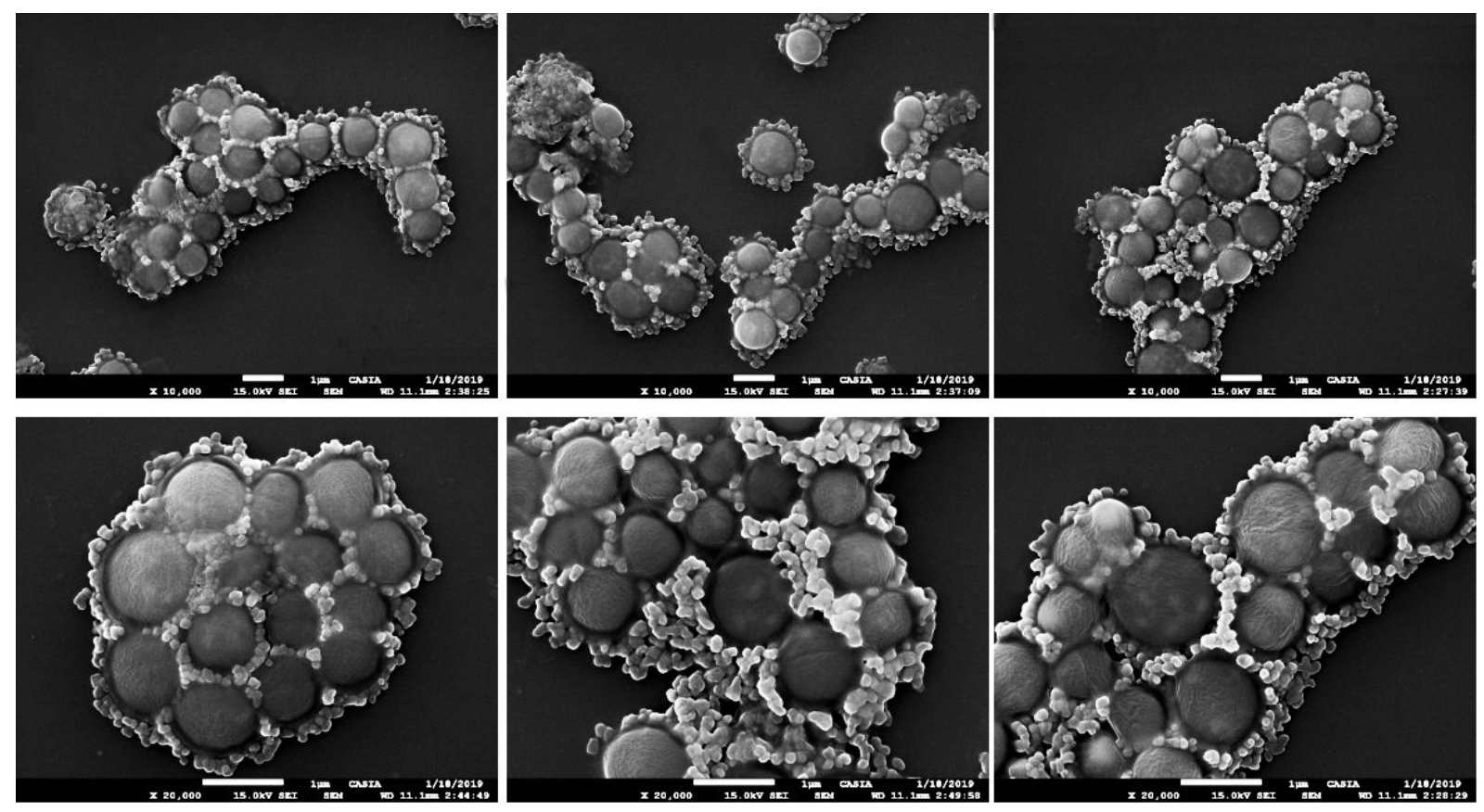

Figure S7. The aggregation and adhesion behavior of BA-BBR NPs on S. aureus observed by FESEM. 

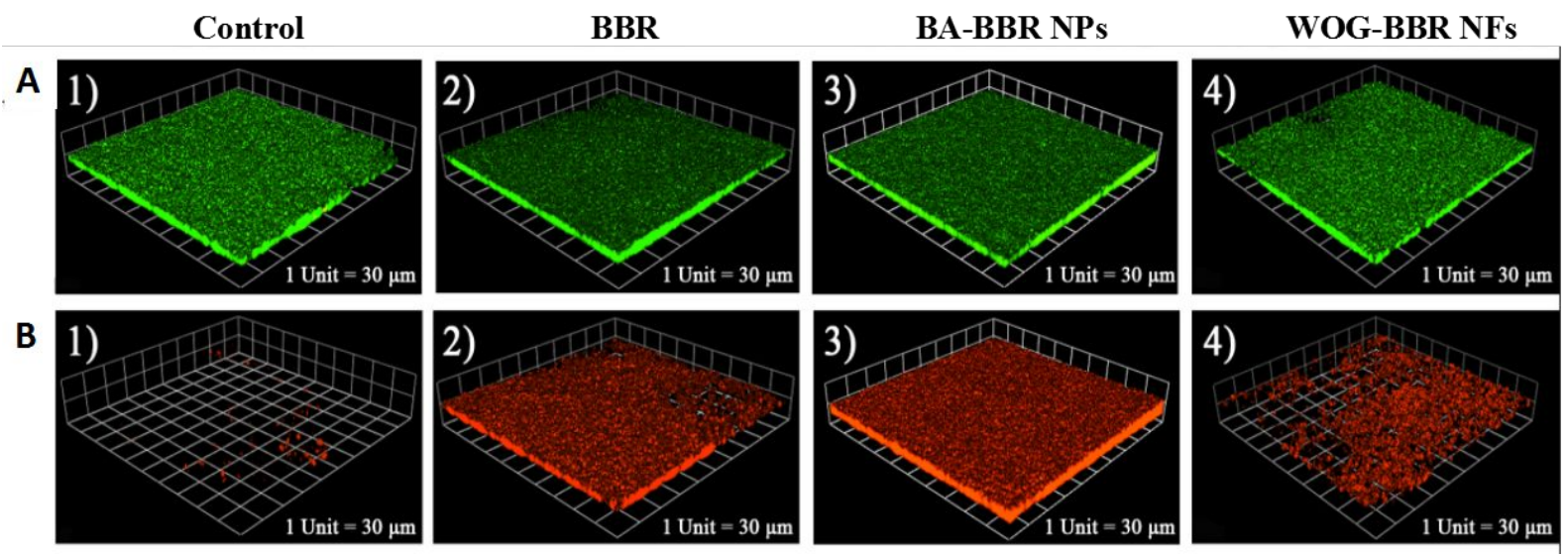

Figure S8. The images of Live/Dead staining assay. (A) Green fluorescence of the Syto9 probe indicated bacterial cells with intact membrane while (B) red fluorescence of Propidium Iodide (PI) indicated dead bacterial. 


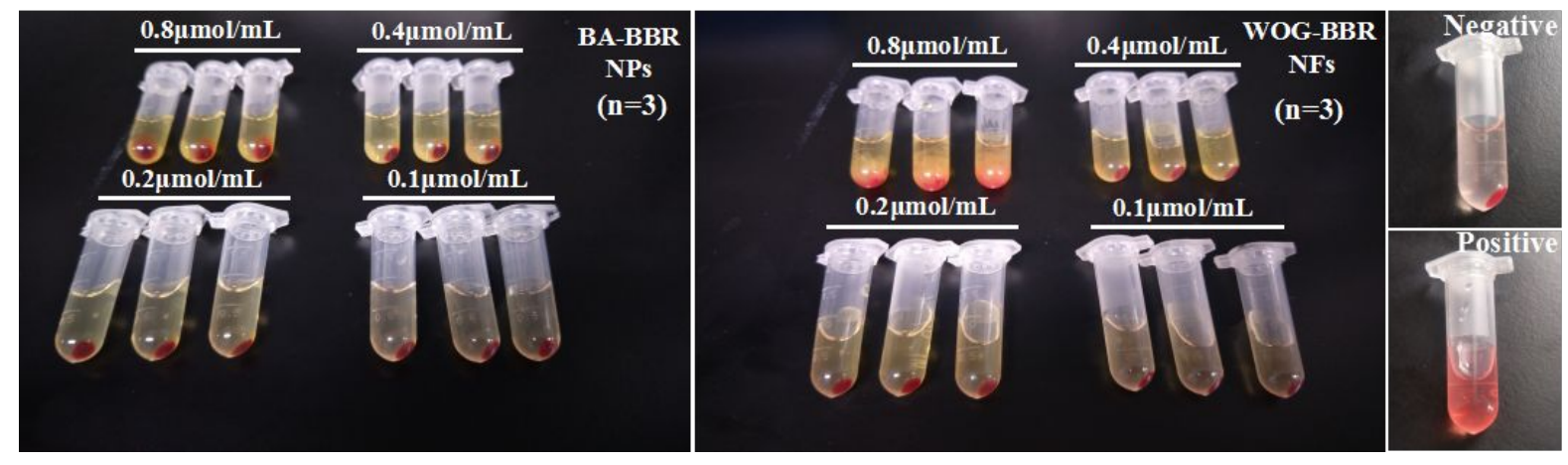

Figure S9. The images of hemolysis test of negative control, positive control, BA-BBR NPs and WOG-BBR NFs. $(\mathrm{n}=3)$ 


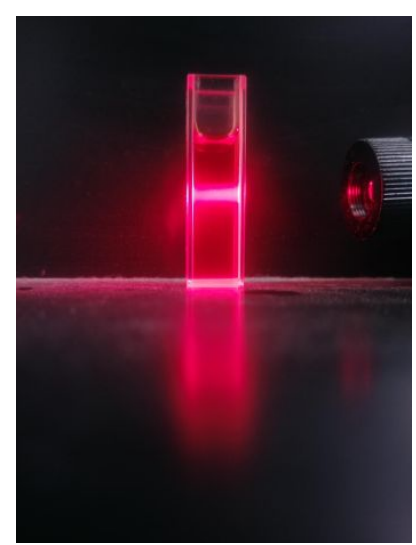

Figure S10. Tindal effect of BA-BBR NPs in deionized water. 